In den 70er- und 80er-Jahren des letzten Jahrhunderts schossen in der durch die Psychiatrieenquete mitbewirkten Reform der psychiatrischen Versorgung psychiatrische Hilfsvereine wie Pilze aus dem Boden. Dieser Gründungsboom setzte sich nach der deutschen Vereinigung in den neuen Ländern fort, sodass es inzwischen in Deutschland keine Versorgungsregion mehr geben dürfte, die nicht über mindestens einen Hilfsverein verfügt. Meistens gibt es sogar mehrere dieser Vereine in der Region, die sich die Trägerschaft der außerklinischen, vor allem der komplementären Dienste und Einrichtungen untereinander aufteilen, wobei sie in der Regel eng mit dem stationären Bereich zusammen arbeiten, von Ausnahmen einmal abgesehen. Vielfach wurden die Vereine aus den Kliniken selbst heraus gegründet. Der Offenbacher Verein Lebensräume e.V. ist hierfür ein gutes Beispiel.

Ohne die lokalen psychiatrischen Hilfsvereine wäre die Psychiatriereform nur Stückwerk geblieben, was sich nicht zuletzt dort beobachten lässt, wo diese nicht flächendeckend vorhanden sind. Betreutes Wohnen, beschütztes Arbeiten, Tagesstätten und eine Vielfalt ambulanter Dienste gehören inzwischen ebenso zum psychiatrischen Weichbild einer Region wie die Fachabteilung am kommunalen Allgemeinkrankenhaus oder das Fachbzw. Sonderkrankenhaus herkömmlicher Struktur in ländlicher Lage und überörtlicher Trägerschaft. Zusammen mit den niedergelassenen Haus- und Nervenärzten sowie den sozialpsychiatrischen Diensten an den Gesundheitsämtern bilden sie ein komplexes Netzwerk und sind die wichtigsten Träger der psychiatrischen Versorgung.

Alle hier aufgeführten Einrichtungen und Dienste sind regelfinanziert. Für alles und jedes gibt es einen Kostenträger, auch wenn es manchmal erhebliche Mühe macht, den wirklich $\mathrm{Zu}$ ständigen herauszufinden. Regelfinanziert heißt aber auch, bezahlt werden die obligatorischen Leistungen, diejenigen, die den Kostenträgern gesetzlich vorgeschrieben sind, für alles wei- tere fühlt sich niemand mehr zuständig. In diese Lücke sind bisher ebenfalls die Vereine eingesprungen, in dem sie „psychiatrische Extras“, einen Patientenausflug oder eine Ferienreise aus ihrem Spendenaufkommen finanzierten oder mitfinanzierten. Auch der Besuch kultureller oder sportlicher Veranstaltungen wurde aus diesem „Topf“ bezahlt. Dies wird sicherlich auch weiterhin der Fall sein, freilich auf einem deutlich niedrigeren $\mathrm{Ni}$ veau.

In dieser Situation kommt es darauf an, neue Geldquellen zu erschließen. Die Gründung einer Stiftung ist eine derartige Geldquelle, und zwar eine, die niemals versiegt. Stiftungen nämlich sind - im Gegensatz zu Vereinen oder „gemeinnützigen GmbHs“ - auf Ewigkeit angelegt, da das von ihnen eingesammelte Kapital nicht angetastet werden darf. Ausgeben kann man nur die Zinsen, abzüglich desjenigen Anteils (max. ein Drittel), der in den Kapitalstock zum Ausgleich der Geldentwertung zurückgeführt werden sollte. Das aber sind schon Details, um die sich der Stiftungsvorstand zu kümmern hat.

Was aber sind die Vorteile einer Psychiatrie-Stiftung im Vergleich zu einem psychiatrischen Hilfsverein, sind beide nicht schließlich Konkurrenten auf dem enger gewordenen Markt um Spenden- und Bußgeldanteile? Gibt es, auf Sicht gesehen, eine vernünftige Arbeitsteilung? Ich denke ja und will dies mit eigenen Erfahrungen auch begründen, da wir in Offenbach inzwischen beides haben, einen mit über 100 fest angestellten Mitarbeitern florierenden Trägerverein „Lebensräume e.V.“ und seit einem Jahr auch eine Stiftung, die Psychiatrie-Stiftung Offenbach. Beides ergänzt sich aufs Trefflichste.

Während die Mitglieder, Freunde und Förderer von „Lebensräume“ ganz überwiegend „psychiatrienahe“ Personen sind, ist dies bei der Stiftung gerade umgekehrt. Die Gründungsstifter sind alle vermögende Persönlichkeiten, die nie und nimmer größere Summen einem „Verein“ zur Verfügung gestellt hätten, nicht zu- 
letzt deswegen, weil sie keinen Einfluss darauf hätten nehmen können, was mit dem Geld geschieht. Als Vorstandsmitglieder der Stiftung können sie das sehr wohl, denn sie selbst entscheiden jährlich, wohin die Mittel fließen sollen. Noch ein weiteres kommt hinzu. In der Stiftungssatzung ist festgelegt, dass neben der Wohlfahrt auch die Kunst und die Wissenschaft gefördert werden können, sofern die künstlerischen und wissenschaftlichen Aktivitäten mittelbar oder unmittelbar dem Wohle psychisch Kranker und ihrer Familien dienen. In diesem Jahr unterstützt die Stiftung deshalb ein „Bildhauerprojekt“ auf dem Klinikgelände, bei dem unter Anleitung zweier Offenbacher Künstler eine Gruppe von Patienten Steine in Skulpturen verwandeln wird. Natürlich wird das Projekt öffentlich begleitet, und, wenn möglich, soll auch ein kleiner Katalog entstehen. Kulturelle Öffentlichkeitsarbeit ist Teil eines gemeindezentrierten Antistigmaprogramms. Eines nicht allzu fernen Tages wird auch die „Wissenschaft" an der Reihe sein, denn auch sie kommt mittelbar und langfristig unseren Patienten zu Gute.

Eine Stiftung zu gründen ist nicht nur für diejenigen interessant, die sich damit „ein Denkmal setzen“ wollen, sondern auch und gerade für Angehörige von Patienten. Ein Beispiel mag dies verdeutlichen. Vor einigen Jahren erbte einer unserer - erfolgreich enthospitalisierten - Langzeitpatienten nach dem Tode seiner Mutter deren Eigentumswohnung, die er für einige Jahre auch bewohnte. Jetzt ist er älter geworden und auch körperlich hinfälliger, sodass er in ein von „Lebensräume“ betriebenes Wohnheim umsiedeln musste. Während er bis dahin mit seiner kleinen Rente und Unterstützung des Betreuten Wohnens sein persönliches Auskommen hatte, wurde er vom Tage der Übernahme ins Wohnheim "Selbstzahler“ - und bleibt dies so lange, bis sein gesamtes Vermögen aus dem Verkaufserlös der Eigentumswohnung aufgebraucht ist. Danach übernimmt der Landeswohlfahrtsverband Hessen als zuständiger Sozialhilfeträger die Kosten. Der zwangsweise Verkauf der Wohnung hätte vermieden werden können, wenn die Mutter ihrem Sohn zwar die Wohnung vererbt, zeitgleich aber die Stiftung in einem notariell gefertigten Behindertentestament als Nacherben eingesetzt hätte. Zwei Fliegen wären so mit einer Klappe geschlagen worden: Der Sohn wäre weder zum zeitweilig vermögenden Selbstzahler und danach zum Sozialhilfeempfänger mit Anspruch auf ein Taschengeld geworden und der Stiftung wäre nach dem Tod des Sohnes das Vermächtnis der Mutter zugefallen.

Auf diese Art und Weise Vermächtnisse zu akquirieren und damit die Wirkungsmöglichkeiten der Stiftung zu vergrößern ist nicht das geringste Motiv, Stiftungen überhaupt zu gründen. Seit dem Bestehen der im Frühjahr 2003 gegründeten Offenbacher Psychiatrie-Stiftung haben bereits mehrere Personen zu erkennen gegeben, dass sie ihr Testament zugunsten der Stiftung geändert haben oder es zu ändern gedenken. Darunter sind auch Angehörige unserer Patienten.

Niemand sollte jedoch glauben, dass wir Psychiater eine Stiftung gründen könnten, um damit anderen Menschen das Geld aus der Tasche zu ziehen. Wir müssen schon bereit sein, einen nicht unbeträchtlichen Teil des Stiftungskapitals selbst aufzubringen, um dann in einem zweiten Schritt Mitstifter zu gewinnen. Das im Jahre 2002 novellierte und modernisierte Stiftungsrecht macht Stiftungen auch unter steuerlichen Gesichtspunkten attraktiver.
So können für Zuwendungen an gemeinnützige Stiftungen bis zu $€ 20450$,- im Jahr steuerlich abgesetzt werden. Zusätzlich können die Stifter bei der Gründung im Jahr bis zu $€ 307000$,pro Person als Sonderausgaben steuerlich geltend machen. Dieser Betrag kann auf einmal in Abzug gebracht werden oder aber verteilt über einen Zeitraum von bis zu zehn Jahren. Diese Summen erscheinen auf den ersten Blick gewaltig, für die anstehende Generation der vor oder im Zweiten Weltkrieg geborenen zukünftigen Erblasser sind sie jedoch nicht so erschreckend hoch wie es auf den ersten Blick aussieht. Dies gilt vor allem für gut situierte, kinderlose Ehepaare (möglichst noch ohne Geschwister und insofern auch ohne Nichten und Neffen), bei denen schon die Frage auftaucht, „wer soll jetzt eigentlich unser Vermögen erben?" Auch deswegen sind Stiftungen in letzter Zeit in Mode gekommen, weil sich mit $€ 100000$,- jedermann ein Denkmal setzen kann. Wenn man sich mit mehreren zusammen tut, ist das keine wirklich große Summe mehr, aber durchaus schon eine kleine Stiftung.

Im vergangenen Jahr wurden in Deutschland rund 1000 Stiftungen neu gegründet, die Gesamtzahl der Stiftungen erhöhte sich damit auf ca. 11000. Ein Drittel davon dient ausschließlich oder in erster Linie sozialen Zwecken. Mit einem Jahresumsatz von $€$ 16 bis 18 Milliarden sind sie inzwischen zu einem bedeutsamen Wirtschaftsfaktor geworden. Etwa $75 \%$ verfügen über ein Stiftungsvermögen von weniger als $€ 500000$,-. In diesem Segment sind auch die wenigen Psychiatrie-Stiftungen angesiedelt, die es derzeit in Deutschland gibt. Wünschenswert wäre natürlich, dass jede psychiatrische Versorgungsregion eine eigene Psychiatriestiftung vorweisen könnte, dann kämen leicht mehr als 300 Stiftungen zusammen. Dies wäre im Übrigen ein bedeutsamer Beitrag zur laufenden Antistigmakampagne und vor allem ein nachhaltiger, denn: Stiftungen sind ewig und ihre Folgen auch.

Wer sich umfassender über das Prozedere der Errichtung einer Stiftung kundig machen will, sei auf den „Bundesverband deutscher Stiftungen“ verwiesen, der im Internet unter www. stiftungen.org firmiert. Dort findet man auch viele brauchbare Literaturhinweise.

Darüber hinaus steht der Unterzeichner mit Rat und eventuell auch Tat für weitere Informationen und praktische Hilfen zur Verfügung. Auch wenn es zunächst nur zu einem Informationsaustausch unter den jetzt bestehenden Psychiatrie-Stiftungen käme, würde dies die Sache voranbringen. Dabei könnte und würde die Psychiatrie-Stiftung Offenbach koordinierende Funktionen gerne wahrnehmen.

Wir bitten daher ausdrücklich um Zuschriften unter der Adresse:

\section{Psychiatrie-Stiftung Offenbach}

Klinikum Offenbach

Starkenburgring 66

63069 Offenbach

E-mail: manfred.bauer@klinikum-offenbach.de

Bankverbindung: Sparkasse Offenbach

Konto-Nr. 1661

BLZ 50550020 